\title{
Open Stabilization Procedures of the Shoulder in the Athlete: Indications, Techniques, and Outcomes
}

\author{
Aryan Haratian (D) \\ Katie Yensen \\ Jennifer A Bell \\ Laith K Hasan \\ Tara Shelby \\ Brandon Yoshida \\ loanna K Bolia (D) \\ Alexander E Weber \\ Frank A Petrigliano $\mathbb{D}$
}

USC Epstein Family Center for Sports Medicine at Keck Medicine of USC, Los Angeles, CA, USA
Correspondence: Frank A Petrigliano USC Epstein Family Center for Sports Medicine at Keck Medicine of USC, 1520 San Pablo Street, Suite 2000, Los Angeles, CA, 90033, USA

Tel +l 323 442-5822

Email fpetrigliano@gmail.com
Abstract: Shoulder instability is a relatively common injury especially in the young athletic population and its surgical management continues to remain a controversial topic in sports medicine orthopedics. Anterior instability is the most common type encountered and is estimated to have an incidence rate of 0.08 per 1000 person-years in the general population; however, this figure is likely higher in the young athletic population. While in recent practice, arthroscopic surgery has become the new gold standard for management, reported failure rates as high as $26 \%$ and high recurrence rates in specific subpopulations such as young men in high collision sports have led to the consideration of alternative open procedures such as open Bankart repair, Latarjet, capsular shift, and glenoid bone grafting. These procedures may be preferred in specific patient subgroups such as young athletes involved in contact sports and those with Hill-Sachs defects and multidirectional instability, with postoperative recurrence rates of instability as low as $10 \%$. The purpose of this review is to provide an overview of different open surgical techniques in the management of shoulder instability and summarize patient outcomes including recurrence rates for shoulder instability, return to sport, range of motion (ROM), muscle strength, and complications either individually by procedure or in comparison with other techniques, with special focus on their impact in the athletic population.

Keywords: open shoulder stabilization, athletes, anterior instability, open Bankart repair, open Latarjet, open capsular shift

\section{Introduction}

The glenohumeral joint allows for 6 degrees of freedom, making it the most mobile joint in the body, as well as the most likely to dislocate. Together with the elbow, it allows us to place our hand in space to perform activities of daily living to more complex movements seen in overhead sports. While the minimal bony restrictions allow the shoulder maximal mobility, the shoulder must rely upon static and dynamic stabilizers for stability. ${ }^{1}$ Static stabilizers include the glenohumeral ligaments and the glenoid labrum. Dynamic stabilizers include the rotator cuff muscles, rotator interval, long head of the biceps and periscapular muscles. ${ }^{2}$

Shoulder instability can be caused by traumatic injuries, atraumatic injuries due to repetitive microtrauma, or from pre-existing generalized ligamentous laxity. As such athletes, especially those involved in contact sports are at particular risk for this injury. Patients presenting with shoulder instability can present with a broad array of symptoms ranging from recurrent dislocation to joint instability and activity-related pain. Anterior instability is the most common, usually due to a fall onto an outstretch arm with the shoulder in abduction and external 
rotation. ${ }^{1}$ A population study has estimated the incidence rate of anterior shoulder dislocation in the general population as 0.08 per 1000 person-years, however this number is likely higher in the young athletic population with estimates of incidence rates of 1.69 per 1000 personyears in US military personnel. ${ }^{3}$ Other less common forms of shoulder instability include posterior and multidirectional instability. ${ }^{4,5}$ Posterior instability is most commonly caused by a traumatic event with the shoulder in flexion, adduction and internal rotation. ${ }^{4}$ Multidirectional instability is defined as symptomatic instability in two or more directions, and most commonly has an insidious onset of nonspecific activity-related pain in the second or third decade of life. ${ }^{5}$ Anterior shoulder instability due to a dislocation event is commonly associated with a HillSachs and/or Bankart lesion. A Hill-Sachs lesion is a compression fracture of the posterosuperolateral humeral head and usually results from anteroinferior glenohumeral dislocation. ${ }^{6}$ Many authors have evaluated the critical glenoid bone loss that is associated with recurrent instability, reporting it to be between $13.5 \%$ to $25 \%$. $^{7-10}$

Evaluating patients with instability symptoms requires a detailed history including dislocations events and prior treatments, mechanism of injury, number of dislocations, occupations and hobbies, and previous surgeries. ${ }^{11}$ A thorough exam of bilateral shoulder including palpation, range of motion (ROM) and neurovascular exam should be performed. Anterior instability exams include anterior load and shift, apprehension and relocation, anterior release and anterior drawer. Posterior instability exams include jerk test, Kim test, posterior load and shift, and posterior drawer. Inferior instability exams include sulcus and gagey test. ${ }^{1,11}$ Calculating a Beighton score can be helpful in identifying patient with generalized laxity. Radiographs including AP, lateral and axillary view should be obtained in all patients. When evaluating for glenoid bone loss and a Hill-Sachs lesions, a West Point axillary view and Stryker notch view, respectively, should be obtained. ${ }^{6}$ Computed tomography (CT) scan is the gold standard for evaluating bone loss, and is pertinent in decision making and preoperative planning. Nonsurgical management of anterior shoulder dislocation includes immobilization and early rehabilitation. ${ }^{1}$ Typically, immobilization can last anywhere from one to two weeks. Rehabilitation focuses on strengthening dynamic stabilizers of the glenohumeral joint. However, there is still controversy regarding the treatment of first-time dislocations as nonsurgical treatment results in higher recurrence rate and may even increase bone loss over time. ${ }^{12}$

The most common treatment for anterior shoulder instability in current practice remains arthroscopic Bankart repair, although failure rates as high as $26 \%$ have been reported. ${ }^{13-17}$ Risk factors for recurrent dislocation following arthroscopic repair include a history of multiple prior dislocations, glenoid bone loss, presence of an off-track Hill-Sachs lesion and the total duration of instability symptoms. ${ }^{18}$ In patients with these characteristic, open stabilization techniques including open Bankart repair, Latarjet, capsular shift, and glenoid bone grafting can be considered as alternatives, that may provide more optimal long term outcomes. The purpose of this review is to summarize open techniques currently used in practice for the management of shoulder instability. An overview of indications, surgical technique and patient outcomes will be provided with special focus to outcomes of these techniques in the athletic population.

\section{Open Bankart Repair}

Bankart repair remains one of the most frequently employed surgical interventions for the management of shoulder instability. The premise of this technique is surgical repair of the torn labrum and shoulder capsule (Bankart lesion), usually accomplished through suture anchor assisted repair and affixing of the capsulolabral complex to the glenoid, thereby restoring shoulder stability. ${ }^{19-21}$ For the open Bankart repair, a deltopectoral approach is typically utilized, with subsequent separation of the deltopectoral interval and the clavipectoral fascia. $^{21,22}$ Typically, a retractor is inserted into the subacromial space to expose the subscapularis. The subscapularis muscle and tendon are incised and split along the middle third, and stay sutures are inserted into the subscapularis to assist with exposure of the shoulder capsule. $^{22}$ A T-Capsulotomy is performed followed by full exposure of the anterior glenoid. ${ }^{21}$ The Bankart lesion is then assessed, and suture anchors are used in a horizontal mattress fashion running to secure the capsulolabral complex to the glenoid, while taking care to avoid injury of the axillary nerve. ${ }^{21,22}$ Based on surgeon preference, a supplemental capsular shift can be used in conjunction with Bankart repair. ${ }^{21}$ Postoperatively, patients are typically placed in a shoulder sling 3 weeks with allowed ROM of hand, wrist, and elbow with limited external rotation of the elbow to 30 degrees for the first 6 weeks. $^{22}$ Average recovery time is typically a minimum 
of 6 months before athletes are allowed to return to contact sports. ${ }^{21,22}$ The open Bankart repair has a high success rate in restoring shoulder stability with recurrence rates estimated to be around $7.7 \%$ after open surgery. ${ }^{23}$ This relatively low recurrence rate suggests high utility for athletes who involved risky contact sports that create a propensity for reinjury and redislocation. ${ }^{22}$ The size of the Bankart lesion can impact rates of recurrence. ${ }^{24}$ The most frequently reported long-term complications include recurrence of instability, loss of ROM, and the development of osteoarthritis potentially necessitating shoulder arthroplasty. ${ }^{25}$ While previously the gold standard in treatment, open Bankart repair is less commonly performed in recent practice with the growing popularity of arthroscopic Bankart surgery. ${ }^{21}$ Nonetheless, open Bankart surgery continues to remain in use, often employed either based on surgeon preference or in cases of recurrent anterior shoulder instability. Additionally, in younger patients who are collision athletes or have accompanying HillSachs lesions, are particularly predisposed to recurrent instability and may benefit from an open Bankart procedure to ensure the highest likelihood of success. ${ }^{26,27}$

\section{Patient Outcomes in Open Bankart Repair}

Numerous studies have explored patient outcomes after open Bankart repair. ${ }^{13,26,28-36}$ Pavlik et al reported on 44 athletes who received open Bankart repair and noted that $88 \%$ were able to return to sports, with $66 \%$ at the same level postoperatively. ${ }^{28}$ Patients who were not able to return to sports $(12 \%)$ reported pain during abduction and external rotation as well as apprehension. ${ }^{28}$ These results have been supported by other studies exploring long term outcomes of open Bankart repair in both the athletic and non, showing recurrence rates as low as $2-6 \%$, and return to sport rates have typically been reported in the 80-90\% range, increased Rowe shoulder scores, however with the drawback of decreased ROM in external and internal rotation, although the majority of patients have no measurable loss of shoulder functionality in relation to decreased ROM. ${ }^{29,36,37}$ Many have reported postoperative Rowe shoulder instability scores $>90$ after open Bankart repair which is promising for athletes seeking to return to sports. ${ }^{36}$ Numerous variations of the open Bankart repair procedure have also been discussed in literature, with recent studies comparing patient outcomes between the different variations in suture techniques such as the use of bioabsorbable staples versus non bioabsorbable sutures. $^{38}$ The Neer modification of the open Bankart procedure involves a supplemental superoinferior capsular shift following labral repair with the intent of providing superior ROM in external rotation versus the classical open Bankart repair, however one study has shown relatively worse rates of return to sport and increased risk of recurrent instability versus open Bankart repair. ${ }^{39,40}$

\section{Comparison of Outcomes in Open Versus Arthroscopic Bankart Repair}

While both open and arthroscopic Bankart repair are used in current practice, multiple studies have aimed to compare outcomes between these two techniques. Rhee et al reported on a cohort of 46 collision athletes, undergoing either arthroscopic or open Bankart repair and noted a higher rate of postoperative instability in those receiving arthroscopy versus an open procedure. ${ }^{13}$ Another study reported better recovery of postoperative muscle strength in patients undergoing arthroscopic Bankart as compared to an open procedure. ${ }^{34}$ These results have been supported to a degree by studies comparing open versus arthroscopic Bankart repair in cases of recurrent shoulder instability concluding that patients undergoing arthroscopic Bankart surgery had improved ROM and muscle strength, however at the cost of increased recurrence of shoulder instability, apprehension, and reoperation than those receiving open Bankart repair. ${ }^{13,26,30-35}$ In cases of recurrent instability, those receiving open Bankart repair have been shown to develop instability, on average, 21.6 months later than those receiving an arthroscopic procedure, thus suggesting that the open procedure provides a more robust and greater longevity of stability than its arthroscopic counterpart. ${ }^{41}$ However, this notion has been challenged by other studies that suggest arthroscopic Bankart repair has recurrence and reoperation rates similar to or in some cases lower than open repair. ${ }^{42-44}$ In the pediatric athletic population, similar rates of recurrence and functional outcomes have been observed in open and arthroscopic Bankart repair, further contrasting from adult studies that frequently report lower recurrence rates in those receiving open Bankart repair. $^{45}$ Interestingly, a small study in older patients, $>50$ years of age, has shown the rates of recurrence between open and arthroscopic Bankart repair have been shown to be comparable, although given the size of this analysis, larger scale studies are needed to better compare these two procedures in older patients. ${ }^{46}$ In cases of failed 
arthroscopic Bankart repair, an open revision Bankart operation can provide reduced rates recurrent shoulder instability and functionality, however with the potential of postoperative loss in forward flexion and external rotation. $^{47,48}$ Cost-analysis studies have indicated that Arthroscopic Bankart repair has a lower total cost than open repair, most predominantly due to the longer operative and post anesthesia care unit time a patient requires after open Bankart repair. ${ }^{49-52}$

\section{Complications of Open Bankart Repair}

The most frequently reported complications in open Bankart repair include loss of ROM predominantly decreased external and internal rotation of the shoulder, increased risk of secondary osteoarthritis necessitating arthroplasty, loss of function of the subscapularis, recurrence of shoulder instability and reoperation. ${ }^{53,54}$ Generally, loss of external and internal rotation is the most common short-term complication associated with open Bankart repair. ${ }^{55}$ Loss of external rotation after Bankart repair has been implicated as a major factor in influencing the rate of patient dissatisfaction, with a previous study in athletes has shown an average loss of 11 degrees after open Bankart repair as compared to the non-injured shoulder, thereby potentially negatively impact athletic performance. ${ }^{55}$ Additionally, proper repair of the subscapularis muscle and tendon must be achieved surgically to provide adequate restoration of muscle function, and has been implicated in influencing patient satisfaction after open Bankart repair. ${ }^{56}$ The estimated risk of additional procedures 1 year postoperatively after Bankart repair has been estimated at around $10 \% .{ }^{53}$ Overall, open Bankart repair is associated with less incidence of complications than other open shoulder stabilization procedures. ${ }^{53}$ Both complication rates and rates of secondary procedures have been shown to be significantly lower in patients receiving open Bankart when compared to open coracoid process transfer and anterior/posterior glenoid bone grafting. ${ }^{53}$

\section{Open Latarjet Technique}

The Latarjet procedure is a transfer procedure that provides a triple blocking effect by using a coracoid bone block to increase surface area of the inferior glenoid fossa, the conjoined tendon to prevent anterior subluxation of the humeral head and the joint capsule to stabilize the structures (Figure 1). ${ }^{57}$ For an open Latarjet procedure, the deltopectoral is often used. ${ }^{58}$ The first part of the procedure involves preparation of the coracoid block in which the coracoid process via soft tissue dissection of the CA ligament, the coracohumeral ligament and the pectoralis minor. $^{58}$ The coracoid processes then harvested using a coracoid osteotomy. ${ }^{59}$ The bone block is then drilled in preparation for implantation. ${ }^{59} \mathrm{Next}$, the glenoid needs to be exposed which can be done through a subscapularis longitudinal split incision along the superior to middle third or a vertical tenotomy followed by a capsulotomy. ${ }^{59,60}$ The coracoid bone block is subsequently positioned flush on the glenoid in which the concave side of the coracoid lines up with the joint surface. ${ }^{59}$ The joint capsule is then
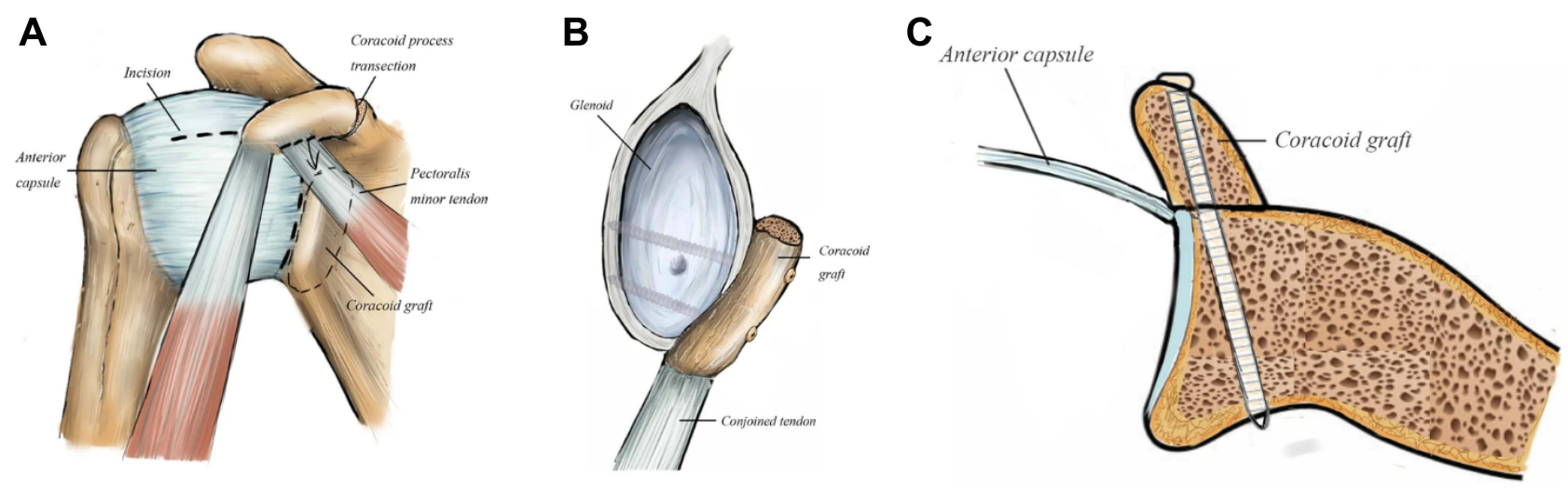

Figure I Schematic of the Latarjet Procedure. (A) An inverted L-shaped opening (dotted line) is made in the anterior approach to form the capsule flap from the glenoid neck. Pectoralis minor (dotted line) is detached from the coracoid before the coracoid osteotomy is carried out. (B) Coracoid graft is fixed to glenoid rim with 2 malleolar screws. If the curve is not fit, the graft can be re-sharpened. (C) Put the graft onto glenoid rim as an extension of the articular platform.

Notes: Adapted from: Xu Y, Wu K, Ma Q et al. Comparison of clinical and PROs of three procedures for recurrent anterior shoulder instability: arthroscopic Bankart repair, capsular shift, and open Latarjet. J Orthop Surg Res. 2019;14(I):326. ${ }^{91}$ Copyright @ 2019 The Authors. Creative Commons Attribution 4.0 International License; CCBY-4.0 (https://creativecommons.org/licenses/by/4.0/legalcode). Modified through adding "Schematic of the Latarjet Procedure" in the figure legend. Retrieved from https:// josr-online.biomedcentral.com/articles/10.1186/s/3018-019-1340-5. 
repaired, and it is reinforced with the coracoacromial ligament. ${ }^{57}$ The end result is an extra-articular coracoid autograft that augments the glenoid fossa and provides improved stability with an increased arc of motion for the humeral head. ${ }^{61}$

\section{Indications and Outcomes of Open Latarjet Surgery}

Open Latarjet procedure is indicated in patients who are high risk for recurrent instability and who have greater than $20 \%$ glenoid bone loss with resorption or with offtrack Hill-Sachs lesions. ${ }^{57}$ Patient characteristics implicated in increasing the risk for recurrent instability include those who participate in contact sports, and patients who are aged 25 or younger. Additional considerations in determining candidacy for open Latarjet include the occurrence of more than three dislocations requiring formal reduction, a demonstrable glenoid or humeral head bone loss warranting bone augmentation or transfer, the presence of a humeral avulsion of the glenohumeral ligament (HAGL) lesion, the presence of hyperlaxity, or the need for a revision stabilization procedure. ${ }^{57}$ Outcomes of the Latarjet procedure show high return to sport percentages in long term follow up studies. ${ }^{62,63}$ Studies by Yapp et al and Elamo et al, authors found a $100 \%$ return to sport rate at 5 -year and 10-year follow up respectively with overall excellent patient reported outcomes (PROs). ${ }^{62,63}$ Chillemi et al conducted a study with a 25 year follow up in which none of the 40 patients reported any episode of redislocation. ${ }^{64}$

Studies have shown that post-operative complications from open Latarjet procedure range from $7-30 \%{ }^{65,66}$ The most common complications include hematoma, recurrent instability, neurological complication, infection, post-operative stiffness, graft/implant failure or malpositioning, bone block nonunion, and osteoarthritis. ${ }^{67} \mathrm{In}$ a study by Scanlon et al, the authors studied 441 patients and found that hematomas were the most common complication experienced by patients who underwent the Latarjet procedure, while there was no recurrent instability or neurological or hardware complications reported among the patients included in the study. ${ }^{68}$ In contrast, Willemont et al reported that graft nonunion resulting in recurrent instability was the main reason for revision surgery with a nonunion resorption rate of $42 \% .{ }^{69}$ A study by Goodloe et al compared surgical complications in patients who underwent Latarjet procedure and those who underwent arthroscopic Bankart repair with findings showing nearly a 10 -fold increase in the risk of surgical complications which included deep surgical site infections, return to operating room within 30 days, and symptomatic VTE in those who got an open Latarjet surgery. ${ }^{70}$ Another study reviewing post-operative complications by Hendy et al looked at 190 patients and reported 15 complications, for a 90-day complication rate of $9.0 \%{ }^{71}$ Graft or hardware failure occurred in 9 patients $(4.7 \%)$ with loosened or broken screws, and 6 required reoperations. Additionally, there were 6 nerve injuries (3.2\%) which most commonly were combined axillary and suprascapular nerve injuries. Scanlon et al reported a lower 90 -day complication rate of $4.3 \%$ in study population of 441 patients finding hematomas being the most common subacute complications. ${ }^{68}$ A study by Mizuno et al looked at long term outcomes from open Latarjet procedures and found that osteoarthritis may develop in about $23 \%$ of cases but most all cases are mild with no stage 4 arthritis observed at 20 years. ${ }^{72}$ Risks for arthritis progression were old age and highlevel sport activity. Similarly, a study by Ernstbrunner et al showed that the open Latarjet reliably restored anterior shoulder stability, however patients had a significant amount of dislocation arthropathy with $37 \%$ of cases showing severe arthritis progression. ${ }^{73}$

\section{Open versus Arthroscopic Latarjet}

With regards to open vs. arthroscopic surgical techniques for the Latarjet procedure, most recent studies have shown that both are viable options for patients with good clinical outcomes. However, some studies report higher redislocation rates in patients treated with arthroscopic Bankart repair. A study by Mohtadi et al published in 2005 compared open and arthroscopic surgery for anterior shoulder instability showed no difference in patient quality of life but did show a higher recurrence rate in patients treated arthroscopically which was most pronounced in younger patients and higher level athletes. ${ }^{74}$ A systemic review by Imam et al compared 7 cohort studies and found that there was a higher risk of dislocation occurrence after arthroscopic Bankart repair with a risk ratio of 2.87 , however there was no difference in post-op complication rate. ${ }^{75}$ Lastly, in a comparative study by Rai et al, authors reported a higher rate of functional satisfaction and a lower operating cost with the open Latarjet procedure compared to arthroscopic Bankart repair. ${ }^{76}$ 


\section{Open Capsular Shift}

While arthroscopic stabilization is the current gold standard in treatment of shoulder instability, reported failure rates as high as $26 \%$ and high recurrence rates in specific subpopulations such as young men in high collision sports and patients with shoulder hyperlaxity, atraumatic or multidirectional instability, have led to the consideration of other alternatives. ${ }^{13-17}$ One such alternative is open capsular shift with the purpose of joint volume reduction, as increased joint volume has been shown to predispose patients to dislocation and failure of surgery (Figure 2). ${ }^{39,77-79}$ Neer and Foster originally proposed the procedure using 40 shoulders with involuntary inferior and multidirectional instability in which other treatments proved ineffective. Success rate was promising with only one shoulder subluxation 7-months postoperatively. ${ }^{39}$ Many techniques have since been proposed. ${ }^{10,15,39,79-84}$ Miller et al compared efficacy of three anterior techniques, humeral-based (group A), glenoidbased (group B), and a central vertical capsular shift (group C) by monitoring capsular volume change in 24 fresh-frozen human cadaver shoulders. ${ }^{79}$ While all three significantly reduced capsular volume $(\mathrm{A}=48.9 \%, \mathrm{~B}=36.8 \%$, and $\mathrm{C}=$ $40.3 \%$ ), authors recommended use of the humeral-based capsular shift, as it led to the largest volume reduction that was significantly higher than the glenoid-based group.

Literature has shown open capsular shift to be a viable and effective option with low recurrence rates, especially in the context of multidirectional injuries. ${ }^{39,85-89}$ Cooper and Brems reported after 43 capsular shifts, $91 \%$ remained stable 2 years post-operatively and reported good patient satisfaction. ${ }^{85}$ None of this cohort were elite athletes, contrasting with a previous study conducted by Altchek et al following 40 patients with involuntary instability who had been injured by an athletic activity. Patients underwent a glenoid-based T-shaped capsulotomy and follow-up times ranged from 2-7 years. All but one reported improved function, none reported pain, and instability occurrence occurred in only 4 of the $42(9.5 \%)$ shoulders studied. ${ }^{86}$ Bigliani et al also followed 68 shoulders in 63 athletic patients that underwent an anterior-inferior capsular shift procedure. While redislocation rate was low at $1.5 \%$, the authors noted that only 5 out of the 10 throwing athletes returned to previous level of activity, with the overall return of the cohort being $75 \% .{ }^{87}$

Multiple comparisons of capsular shift with other procedures have been made. ${ }^{88-91}$ Ilyes et al compared open capsular shift to non-operative treatments to model muscle activity during pull, push, and upper extremity elevation and overhead throw movement in 34 patients with multidirectional instability. ${ }^{88}$ While similar muscle pattern was shown between the two groups $(p=0.19)$, only capsular shift allowed for repair of ligamentous and labral abnormalities. $^{88}$ Longo et al conducted a systematic review that included 24 studies covering various techniques for multidirectional shoulder instability, finding open capsular shift and arthroscopic capsular plication as the two most effective treatment options with re-dislocation in 17 of $226(7.5 \%)$ shoulders in the former group and 21 of 268 (7.8\%) shoulders in the latter. ${ }^{89}$ Cohen et al found in an in vitro comparison of 15 fresh-frozen human shoulders that underwent either capsular plication or open capsular
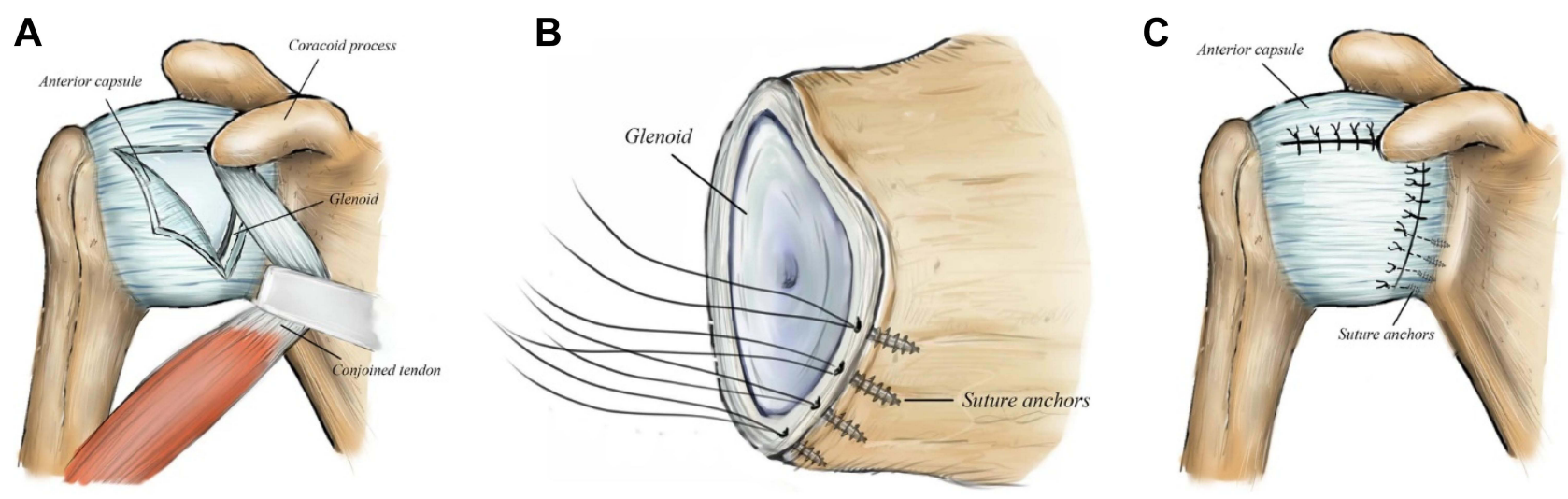

Figure 2 Schematic of Capsular Shift. (A) The capsule is incised based on inverted L shape to expose glenoid rim adequately. (B) Three to four suture anchors are positioned medially from 3 o'clock to 6 o'clock to the direction. (C) Pull the flap superiorly to make incised capsule tied down to the glenoid edge.

Notes: Adapted from: Xu Y, Wu K, Ma Q et al. Comparison of clinical and PROs of three procedures for recurrent anterior shoulder instability: arthroscopic Bankart repair, capsular shift, and open Latarjet. J Orthop Surg Res. 2019;14(I):326. ${ }^{91}$ Copyright (C 2019 The Authors. Creative Commons Attribution 4.0 International License; CCBY-4.0 (https://creativecommons.org/licenses/by/4.0/legalcode). Modified through adding "Schematic of Capsular Shift" in the figure legend. Retrieved from https://josr-online. biomedcentral.com/articles/10.1186/s 13018-019-1340-5. 
shift, $22.8 \%$ volume reduction in the former $49.9 \%$ in the latter. Jacobson et al also compared arthroscopic capsular plication to open capsular shift, finding comparable return to sport and recurrence events, with overall complications being low in both groups. ${ }^{90} \mathrm{Xu}$ et al compared three techniques, arthroscopic Bankart, open Latarjet, and capsular shift, with Rowe scores of $92.3 \pm 1.5,96.2 \pm 2.1$, and $93.2 \pm 2.3$ that significantly differ and no difference in patient satisfaction. ${ }^{91}$ One patient in each the Bankart and capsular shift groups had post-operative recurrence. ${ }^{91}$ The authors make the suggestion of capsular shift use for multidirectional instability, while Bankart had rapid recovery and Latarjet provided greater stability with a lower recurrence rate. ${ }^{91}$

\section{Anterior/Posterior Bone Grafting}

Autograft or allograft bone grafting for the treatment of anterior or posterior shoulder instability is a novel technique whose adoption is rapidly increasing. This procedure can be performed either open or arthroscopically and achieves increased stability by increasing the glenoid surface area. ${ }^{92-98}$ Via either modality, bone graft for the treatment of anterior shoulder instability requires fixation of the graft flush to the anterior glenoid rim on the anterior glenoid neck $;^{99}$ for posterior shoulder instability, the graft must either flush or $1 \mathrm{~mm}$ lateral to the native glenoid on the posterior glenoid neck. ${ }^{94}$ Numerous autografts and allografts have been studied ${ }^{94,99-101}$ but distal tibial allograft specifically has been demonstrated to recreate an anatomic reconstruction of the glenoid cavity to reduce early symptomatic glenohumeral arthritis. ${ }^{102}$ One systematic review by Gilat et al showed that autograft vs. allograft bone grafting had similar efficacy and safety. ${ }^{103}$

Indications for bone grafting for anterior shoulder instability: ${ }^{99}$

1. Isolated anterior glenoid bone loss between 20-25\%

2. Anterior glenoid bone less $<20 \%$ with associated Bankart lesion

3. Anterior glenoid bone loss $>10 \%$ but $<20 \%$ with ISIS score of 3-6 points

4. First episode of dislocation within 3 years

5. $\leq 5$ episodes of dislocation

Indications for bone grafting for posterior shoulder instability are, however, less well defined but may be considered in patients with: ${ }^{93,104}$
1. increased glenoid retroversion, previous failed posterior soft-tissue repair, and insufficient posterior capsulolabral tissue

2. Poor-quality posterior capsular tissue

3. Significant posterior laxity

4. Failed previous soft-tissue stabilization with recurrent instability

5. Posterior instability with glenoid dysplasia

6. Traumatic posterior bony Bankart lesion $(<15 \%)$

7. Large reverse Hill-Sachs lesion

8. Bone loss $\geq 25 \%$ the width of the inferior glenoid

Outcome data associated with bone grafting for anterior shoulder instability is overall positive. One systematic review by Gilat et al demonstrated that bone grafting had a rate of recurrent instability (3\%), osteoarthritis $(12 \%)$, other complications $(5 \%)$ a return to sports $(88 \%)$ that was not significantly different to patients who underwent the Latarjet procedure; PRO data was also similar with both procedures demonstrating a significant improvement in American Shoulder and Elbow Surgeons score amongst other PROs. ${ }^{105}$ Outcome data for bone grafting for posterior shoulder instability is less abundant but a study by Meuffels et al showed that at a median of 18 years, 6 of 11 patients were satisfied with their procedure and would elect to have the intervention again. ${ }^{106}$ Seven of 11 had recurrent instability and 4 patients had a recurrent episode of dislocation. However, other literature demonstrates positive outcomes after shorter follow-up ${ }^{92}$ with one study by Schwartz et al showing a significant improvement in Rowe score and Walch-Duplay score from 18.4 to 82.1 points and 37.4 to 82.9 points, respectively $(\mathrm{P}<0.01){ }^{107}$

Complications after anterior or posterior glenoid bone grafting include hardware failure, painful hardware, osteoarthritis, $^{92}$ donor site pain (if autograft was harvested), ${ }^{105}$ and specifically brachial plexus injury in anterior bone graft placement. ${ }^{100}$ Kowalski et al demonstrated in a large Pearldiver database study that both anterior and posterior glenoid bone graft had an overall complication rate (dislocation, closed adhesion release, or reoperation) of approximately $20 \%$, which was significantly greater than Bankart repair. ${ }^{53}$

\section{Conclusion}

Shoulder instability is a relatively common injury especially in the young athletic population and its surgical management continues to remain a controversial topic in sports medicine orthopedics. While in recent practice, arthroscopic surgery has become the new gold standard for management, open 
procedures such as open Bankart repair, Latarjet, capsular shift, and glenoid bone grafting can serve as alternatives and may potentially lead to decreased recurrence rates or be preferred in specific patient subgroups such as young athletes involved in contact sports and those with Hill-Sachs defects and multidirectional instability. Among open procedures, recurrence rates have been comparable and often reported to be below $10 \%$. Open Bankart repair is associated with the lowest rates of postoperative complications and less risk for a secondary procedure as compared to coracoid transfers and bone grafting. As the role of open surgery in the management of shoulder instability continues to evolve, future studies can explore and provide insight to optimize patient selection for open procedures over arthroscopy, and guide surgeons in clinical decision-making.

\section{Disclosure}

The authors report no conflicts of interest for this work and have no financial disclosures to declare.

\section{References}

1. Provencher MT, Midtgaard KS, Owens BD, Tokish JM. Diagnosis and management of traumatic anterior shoulder instability. J Am Acad Orthop Surg. 2021;29(2):e51-e61. doi:10.5435/JAAOS-D-20-00202

2. Farrar NG, Malal JJ, Fischer J, Waseem M. An overview of shoulder instability and its management. Open Orthop J. 2013;7:338-346. doi:10.2174/1874325001307010338

3. Owens BD, Dawson L, Burks R, Cameron KL. Incidence of shoulder dislocation in the United States military: demographic considerations from a high-risk population. J Bone Joint Surg Am. 2009;91(4):791-796. doi:10.2106/JBJS.H.00514

4. Millett PJ, Clavert P, Hatch GF 3rd, Warner JJ. Recurrent posterior shoulder instability. J Am Acad Orthop Surg. 2006;14 (8):464-476. doi:10.5435/00124635-200608000-00004

5. Gaskill TR, Taylor DC, Millett PJ. Management of multidirectional instability of the shoulder. $J$ Am Acad Orthop Surg. 2011;19(12):758-767. doi:10.5435/00124635-201112000-00006

6. Provencher MT, Frank RM, Leclere LE, et al. The Hill-Sachs lesion: diagnosis, classification, and management. $\mathrm{J} \mathrm{Am} \mathrm{Acad}$ Orthop Surg. 2012;20(4):242-252. doi:10.5435/JAAOS-20-04242

7. Dickens JF, Owens BD, Cameron KL, et al. The effect of subcritical bone loss and exposure on recurrent instability after arthroscopic bankart repair in intercollegiate American football. Am J Sports Med. 2017;45(8):1769-1775. doi:10.1177/036354 6517704184

8. Shin SJ, Kim RG, Jeon YS, Kwon TH. Critical value of anterior glenoid bone loss that leads to recurrent glenohumeral instability after arthroscopic bankart repair. Am J Sports Med. 2017;45 (9):1975-1981. doi:10.1177/0363546517697963

9. Lo IK, Parten PM, Burkhart SS. The inverted pear glenoid: an indicator of significant glenoid bone loss. Arthroscopy. 2004;20 (2):169-174. doi:10.1016/j.arthro.2003.11.036

10. Gerber C, Nyffeler RW. Classification of glenohumeral joint instability. Clin Orthop Relat Res. 2002;400:65-76. doi:10.1097/ 00003086-200207000-00009
11. Hippensteel KJ, Brophy R, Smith MV, Wright RW. Comprehensive review of provocative and instability physical examination tests of the shoulder. J Am Acad Orthop Surg. 2019;27(11):395-404. doi:10.5435/JAAOS-D-17-00637

12. Dickens JF, Slaven SE, Cameron KL, et al. Prospective evaluation of glenoid bone loss after first-time and recurrent anterior glenohumeral instability events. Am J Sports Med. 2019;47 (5):1082-1089. doi:10.1177/0363546519831286

13. Rhee YG, Ha JH, Cho NS. Anterior shoulder stabilization in collision athletes: arthroscopic versus open Bankart repair. Am J Sports Med. 2006;34(6):979-985.

14. Tokish JM, Lafosse L, Giacomo GD, Arciero R. Patients in whom arthroscopic bankart repair is not enough: evaluation and management of complex anterior glenohumeral instability. Instr Course Lect. 2017;66:79-89.

15. Carlson Strother CR, McLaughlin RJ, Krych AJ, Sanchez-Sotelo J, Camp CL. Open shoulder stabilization for instability: anterior labral repair with capsular shift. Arthrosc Tech. 2019;8(7):e749e754. doi:10.1016/j.eats.2019.03.011

16. Chan AG, Kilcoyne KG, Chan S, Dickens JF, Waterman BR. Evaluation of the Instability Severity Index score in predicting failure following arthroscopic Bankart surgery in an active military population. $J$ Shoulder Elbow Surg. 2019;28(5):e156-e163. doi:10.1016/j.jse.2018.11.048

17. Balg F, Boileau P. The instability severity index score. A simple pre-operative score to select patients for arthroscopic or open shoulder stabilisation. $J$ Bone Joint Surg Br. 2007;89 (11):1470-1477. doi:10.1302/0301-620X.89B11.18962

18. Lee SH, Lim KH, Kim JW. Risk factors for recurrence of anterior-inferior instability of the shoulder after arthroscopic bankart repair in patients younger than 30 years. Arthroscopy. 2018;34(9):2530-2536. doi:10.1016/j.arthro.2018.03.032

19. Rashid MS, Arner JW, Millett PJ, Sugaya H, Emery R. The Bankart repair: past, present, and future. J Shoulder Elbow Surg. 2020;29(12):e491-e498. doi:10.1016/j.jse.2020.06.012

20. Randelli P, Ragone V, Carminati S, Cabitza P. Risk factors for recurrence after Bankart repair a systematic review. Knee Surg Sports Traumatol Arthrosc. 2012;20(11):2129-2138. doi:10.1007/ s00167-012-2140-1

21. Merrill CAA, Arciero R. Open bankart repair: a reproducible technique. Oper Tech Sports Med. 2019;27(1):42-48. doi:10.1053/j.otsm.2019.01.007

22. Coughlin RP, Crapser A, Coughlin K, Coughlin LP. Open Bankart revisited. Arthrosc Tech. 2017;6(1):e233-e237. doi:10.1016/j. eats.2016.09.027

23. Rollick NC, Ono Y, Kurji HM, et al. Long-term outcomes of the Bankart and Latarjet repairs: a systematic review. Open Access J Sports Med. 2017;8:97-105. doi:10.2147/OAJSM. $\mathrm{S} 106983$

24. Lai DM, Ma H-L, Hung S-C, Chen T-H, Wu -J-J. Bankart repair with suture anchors for traumatic recurrent anterior shoulder instability: comparison of results between small and large Bankart lesions. Knee Surg, Sports Traumatol, Arthroscopy. 2006;14(1):82-87. doi:10.1007/s00167-004-0615-4

25. Pelet S, Jolles BM, Farron A. Bankart repair for recurrent anterior glenohumeral instability: results at twenty-nine years' follow-up. J Shoulder Elbow Surg. 2006;15(2):203-207. doi:10.1016/j.jse.20 05.06.011

26. Mohtadi NG, Chan DS, Hollinshead RM, et al. A randomized clinical trial comparing open and arthroscopic stabilization for recurrent traumatic anterior shoulder instability: two-year follow-up with disease-specific quality-of-life outcomes. $J$ Bone Joint Surg Am. 2014;96(5):353-360. doi:10.2106/JBJS.L.01656

27. Delos DM, Moran C, Warren R. Open bankart repair in contact athletes: why and how. Oper Tech Sports Med. 2013;21 (4):220-224. doi:10.1053/j.otsm.2013.08.007 
28. Pavlik A, Csepai D, Hidas P, Banoczy A. Sports ability after Bankart procedure in professional athletes. Knee Surg Sports Traumatol Arthrosc. 1996;4(2):116-120. doi:10.1007/BF01477 264

29. Neviaser RJB, Neviaser MT. Mid-term to long-term outcome of the open Bankart repair for recurrent traumatic anterior dislocation of the shoulder. J Shoulder Elbow Surg. 2017;26 (11):1943-1947. doi:10.1016/j.jse.2017.04.013

30. Chen L, Xu Z, Peng J, Xing F, Wang H, Xiang Z. Effectiveness and safety of arthroscopic versus open Bankart repair for recurrent anterior shoulder dislocation: a meta-analysis of clinical trial data. Arch Orthop Trauma Surg. 2015;135(4):529-538. doi:10.10 07/s00402-015-2175-0

31. Moroder PO, Pizzinini M, Demetz S, Resch E, Moroder H, Open Bankart P. Repair for the treatment of anterior shoulder instability without substantial osseous glenoid defects results after a minimum follow-up of twenty years. J Bone Joint SurgAm Volume. 2015;97A(17):1398-1405. doi:10.2106/JBJS.N.01 214

32. Uchiyama YH, Handa A, Shimpuku E, Omi H, Hashimoto H, Imai T. Open bankart repair plus inferior capsular shift versus arthroscopic bankart repair without augmentations for traumatic anterior shoulder instability: a prospective study. J Orthopaed Surg. 2017;25(3):230949901772794. doi:10.1177/23094990177 27947

33. Wang LL, Liu Y, Su X, Liu S. A meta-analysis of arthroscopic versus open repair for treatment of bankart lesions in the shoulder. Med Sci Monitor. 2015;21:3028-3035. doi:10.12659/MSM 894346

34. Rhee YG, Lim CT, C S. Muscle strength after anterior shoulder stabilization: arthroscopic versus open bankart repair. Am J Sports Med. 2007;35(11):1859-1864.

35. Lutzner JK, Krummenauer F, Lübke J, Kirschner S, Günther K-P. Functional outcome after open and arthroscopic bankart repair for traumatic shoulder instability. Eur J Med Res. 2009;14(1):18-24. doi:10.1186/2047-783X-14-1-18

36. Hatch $\mathrm{MDH}, \mathrm{L}$ W. The open bankart repair for traumatic anterior shoulder instability in teenage athletes. $J$ Pediatr Orthopaed. 2018;38(1):27-31. doi:10.1097/BPO.0000000000000744

37. Steinbeck JJ, Jerosch J. The open Bankart repair using suture anchors in posttraumatic shoulder instability: 2- to 5-year results. Unfallchirurg. 1997;100(12):938-942. doi:10.1007/s0011 30050215

38. Reuther FM. Open Bankart procedure for shoulder stabilization using bioabsorbable staples. Technique and results. Unfallchirurg. 2004;107(2):99-103. doi:10.1007/s00113-003-0719-1

39. Neer CS 2nd, Foster CR. Inferior capsular shift for involuntary inferior and multidirectional instability of the shoulder. A preliminary report. $J$ Bone Joint Surg Am. 1980;62 (6):897-908. doi:10.2106/00004623-198062060-00004

40. Boileau PF, Bicknell E. Neer modification of open bankart procedure: what are the rates of recurrent instability, functional outcome, and arthritis? shoulder. Clin Orthop Relat Res. 2012;470 (9):2554-2560. doi:10.1007/s11999-012-2296-5

41. Virk MSM, Cote RL, Ware M, et al. Comparison of time to recurrence of instability after open and arthroscopic bankart repair techniques. Orthopaed J Sports Med. 2016;4(6). doi:10.1177/ 2325967116654114

42. Petrera M, Patella V, Patella S, Theodoropoulos J. A meta-analysis of open versus arthroscopic Bankart repair using suture anchors. Knee Surg Sports Traumatol Arthrosc. 2010;18 (12):1742-1747. doi:10.1007/s00167-010-1093-5

43. Tjoumakaris FPA, Hasan JA, Ramsey SA, Williams ML. Arthroscopic and open Bankart repairs provide similar outcomes. Clin Orthop Relat Res. 2006;446(446):227-232. doi:10.1097/01.blo.0000205883.73705.19
44. Zaffagnini SM, Giordano GMM, Bonanzinga G, et al. Long-term outcomes after repair of recurrent post-traumatic anterior shoulder instability: comparison of arthroscopic transglenoid suture and open Bankart reconstruction. Knee Surg, Sports Traumatol, Arthroscopy. 2012;20(5):816-821. doi:10.1007/s00167-011-1674-y

45. Shymon SJR, Edmonds J. Traumatic anterior instability of the pediatric shoulder: a comparison of arthroscopic and open Bankart repairs. J Pediatr Orthopaed. 2015;35(1):1-6. doi:10.10 97/BPO.0000000000000215

46. Sperling JWD, Torchia SFM, O'Driscoll ME, Cofield SW. Bankart repair in patients aged fifty years or greater: results of arthroscopic and open repairs. J Shoulder Elbow Surg. 2005;14 (2):111-113. doi:10.1016/j.jse.2004.06.011

47. Cho NS, Yi JW, Lee BG, Rhee YG. Revision open Bankart surgery after arthroscopic repair for traumatic anterior shoulder instability. Am J Sports Med. 2009;37(11):2158-2164. doi:10.11 77/0363546509339015

48. Neviaser ASB, Neviaser MT, Open RJ. Bankart repair for revision of failed prior stabilization: outcome analysis at a mean of more than 10 years. J Shoulder Elbow Surg. 2015;24(6):897-901. doi:10.1016/j.jse.2014.11.036

49. Barber FA, Click SD, Weideman CA. Arthroscopic or open Bankart procedures: what are the costs? Arthroscopy. 1998;14 (7):671-674. doi:10.1016/S0749-8063(98)70092-1

50. Wang CG, Zarins N, Warner B. Arthroscopic versus open bankart repair: analysis of patient subjective outcome and cost. Arthroscopy - J Arthroscopic Related Surg. 2005;21(10):12 19-1222. doi:10.1016/j.arthro.2005.07.004

51. Bokshan SL-D, Owens SF. Comparison of 30-day morbidity and mortality after arthroscopic bankart, open bankart, and Latarjet-Bristow procedures: a review of 2864 cases. Orthopaed J Sports Med. 2017;5(7):232596711771316. doi:10.1177/2325 967117713163

52. Green MR, Christensen KP. Arthroscopic versus open Bankart procedures: a comparison of early morbidity and complications. Arthroscopy. 1993;9(4):371-374. doi:10.1016/S0749-8063(05) 80308-1

53. Kowalski TJ, Khan AZ, Cohen JR, et al. Open shoulder stabilization: current trends and 1-year postoperative complications. JSES Open Access. 2017;1(2):72-78. doi:10.1016/j.jses.2017.07.001

54. Huerta A, Rincón G, Peidro L, Combalia A, Sastre S. Controversies in the surgical management of shoulder instability: open vs arthroscopic procedures. Open Orthop J. 2017; 11:875-881. doi:10.2174/1874325001711010875

55. Rahme HV, Ludvigsson O, Elven L, Michaelsson M. Loss of external rotation after open Bankart repair: an important prognostic factor for patient satisfaction. Knee Surg Sports Traumatol Arthroscopy. 2010;18 (3):404 408. doi:10.1007/s00167-009-0987-6

56. Sachs RAW, Stone B, Paxton ML, Kuney L. Open Bankart repair - correlation of results with postoperative subscapularis function. Am J Sports Med. 2005;33(10):1458-1462. doi:10.1177/036354 6505275350

57. Fabricant PD, Taylor SA, McCarthy MM, et al. Open and arthroscopic anterior shoulder stabilization. JBJS Rev. 2015;3(2). doi:10.2106/JBJS.RVW.N.00060.

58. Doursounian L, Debet-Mejean A, Chetboun A, Nourissat G. Bristow-Latarjet procedure with specific instrumentation: study of 34 cases. Int Orthop. 2009;33(4):1031-1036. doi:10.1007/ s00264-008-0606-z

59. de Beer JF, Roberts C. Glenoid bone defects-open latarjet with congruent arc modification. Orthop Clin North Am. 2010;41 (3):407-415. doi:10.1016/j.ocl.2010.02.008

60. Aurich M, Hofmann GO, Best N. Clinical outcome and return to sports activity after surgical treatment for recurrent shoulder instability with a modified Latarjet procedure. Orthop Traumatol Surg Res. 2021;107:102977. doi:10.1016/j.otsr.2021.102977 
61. White AE, Patel NK, Hadley CJ, Dodson CC. An algorithmic approach to the management of shoulder instability. $J$ Am Acad Orthop Surg Glob Res Rev. 2019;3(12). e19.00168.

62. Yapp LZ, Nicholson JA, McCallum C, Macdonald DJ, Robinson CM. Latarjet as a primary and revision procedure for anterior shoulder instability - A comparative study of survivorship, complications and functional outcomes in the medium to long-term. Shoulder Elbow. 2020;12(5):338-348. doi:10.1177/ 1758573219864926

63. Elamo S, Selanne L, Lehtimaki K, et al. Bankart versus Latarjet operation as a revision procedure after a failed arthroscopic Bankart repair. JSES Int. 2020;4(2):292-296. doi:10.1016/j. jseint.2020.01.004

64. Chillemi C, Guerrisi M, Paglialunga C, Salate Santone F, Osimani M. Latarjet procedure for anterior shoulder instability: a 24-year follow-up study. Arch Orthop Trauma Surg. 2021;141 (2):189-196. doi:10.1007/s00402-020-03426-2

65. Colegate-Stone TJ, van der Watt C, de Beer JF. Evaluation of functional outcomes and complications following modified Latarjet reconstruction in athletes with anterior shoulder instability. Shoulder Elbow. 2015;7(3):168-173. doi:10.1177/1758573215578588

66. Griesser MJ, Harris JD, McCoy BW, et al. Complications and re-operations after Bristow-Latarjet shoulder stabilization: a systematic review. J Shoulder Elbow Surg. 2013;22 (2):286-292. doi:10.1016/j.jse.2012.09.009

67. Gupta A, Delaney R, Petkin K, Lafosse L. Complications of the Latarjet procedure. Curr Rev Musculoskelet Med. 2015;8 (1):59-66. doi:10.1007/s12178-015-9258-y

68. Scanlon JP, Hurley ET, Davey MS, et al. 90-day complication rate after the latarjet procedure in a high-volume center. Am J Sports Med. 2020;48(14):3467-3471. doi:10.1177/0363546520964488

69. Willemot L, De Boey S, Van Tongel A, Declercq G, De Wilde L, Verborgt $\mathrm{O}$. Analysis of failures after the Bristow-Latarjet procedure for recurrent shoulder instability. Int Orthop. 2019;43 (8):1899-1907. doi:10.1007/s00264-018-4105-6

70. Goodloe JB, Traven SA, Johnson CA, Woolf SK, Nutting JT, Slone HS. Increased risk of short-term complications and venous thromboembolism in Latarjet-Bristow procedures compared with bankart repairs. Arthroscopy. 2021;37(3):806-813. doi:10.1016/j. arthro.2020.10.039

71. Hendy BA, Padegimas EM, Kane L, et al. Early postoperative complications after Latarjet procedure: a single-institution experience over 10 years. J Shoulder Elbow Surg. 2021;30(6):e300e308. doi:10.1016/j.jse.2020.09.002

72. Mizuno N, Denard PJ, Raiss P, Melis B, Walch G. Long-term results of the Latarjet procedure for anterior instability of the shoulder. J Shoulder Elbow Surg. 2014;23(11):1691-1699. doi:10.1016/j.jse.2014.02.015

73. Ernstbrunner L, Wartmann L, Zimmermann SM, Schenk P, Gerber C, Wieser K. Long-term results of the open latarjet procedure for recurrent anterior shoulder instability in patients older than 40 years. Am J Sports Med. 2019;47(13):3057-3064. doi:10.1177/0363546519872501

74. Mohtadi NG, Bitar IJ, Sasyniuk TM, Hollinshead RM, Harper WP. Arthroscopic versus open repair for traumatic anterior shoulder instability: a meta-analysis. Arthroscopy. 2005;21 (6):652-658. doi:10.1016/j.arthro.2005.02.021

75. Imam MA, Shehata MSA, Martin A, et al. Bankart repair versus latarjet procedure for recurrent anterior shoulder instability: a systematic review and meta-analysis of 3275 shoulders. Am $J$ Sports Med. 2021;49(7):1945-1953. doi:10.1177/0363546520 962082

76. Rai S, Tamang N, Sharma LK, et al. Comparative study of arthroscopic Bankart repair versus open Latarjet procedure for recurrent shoulder dislocation. J Int Med Res. 2021;49 (4):3000605211007328. doi:10.1177/03000605211007328
77. Reeves B. Arthrography of the shoulder. J Bone Joint Surg Br. 1966;48(3):424-435. doi:10.1302/0301-620X.48B3.424

78. Duncan R, Savoie FH 3rd. Arthroscopic inferior capsular shift for multidirectional instability of the shoulder: a preliminary report. Arthroscopy. 1993;9(1):24-27. doi:10.1016/S0749-8063(05)80340-8

79. Miller MD, Larsen KM, Luke T, Leis HT, Plancher KD. Anterior capsular shift volume reduction: an in vitro comparison of 3 techniques. $J$ Shoulder Elbow Surg. 2003;12(4):350-354. doi:10.1016/S1058-2746(02)86805-7

80. Yamaguchi K, Flatow EL. Management of multidirectional instability. Clin Sports Med. 1995;14(4):885-902. doi:10.1016/ S0278-5919(20)30186-1

81. Luke TA, Rovner AD, Karas SG, Hawkins RJ, Plancher KD. Volumetric change in the shoulder capsule after open inferior capsular shift versus arthroscopic thermal capsular shrinkage: a cadaveric model. J Shoulder Elbow Surg. 2004;13(2):146-149. doi:10.1016/j.jse.2003.11.008

82. Caprise PA Jr, Sekiya JK. Open and arthroscopic treatment of multidirectional instability of the shoulder. Arthroscopy. 2006;22 (10):1126-1131. doi:10.1016/j.arthro.2006.08.002

83. Wiater JM, Vibert BT. Glenohumeral joint volume reduction with progressive release and shifting of the inferior shoulder capsule. $J$ Shoulder Elbow Surg. 2007;16(6):810-814. doi:10.1016/j.jse. 2007.02.117

84. Peebles LA, Aman ZS, Preuss FR, et al. Multidirectional shoulder instability with bone loss and prior failed latarjet procedure: treatment with fresh distal tibial allograft and modified T-Plasty open capsular shift. Arthrosc Tech. 2019;8(5):e459-e464. doi:10.1016/j.eats.2019.01.002

85. Cooper RA, Brems JJ. The inferior capsular-shift procedure for multidirectional instability of the shoulder. J Bone Joint Surg Am. 1992;74 (10):1516-1521. doi:10.2106/00004623-199274100-00010

86. Altchek DW, Warren RF, Skyhar MJ, Ortiz G. T-plasty modification of the Bankart procedure for multidirectional instability of the anterior and inferior types. J Bone Joint Surg Am. 1991;73 (1):105-112. doi:10.2106/00004623-199173010-00013

87. Bigliani LU, Kurzweil PR, Schwartzbach CC, Wolfe IN, Flatow EL. Inferior capsular shift procedure for anterior-inferior shoulder instability in athletes. Am J Sports Med. 1994;22 (5):578-584. doi:10.1177/036354659402200502

88. Illyes A, Kiss J, Kiss RM. Electromyographic analysis during pull, forward punch, elevation and overhead throw after conservative treatment or capsular shift at patient with multidirectional shoulder joint instability. J Electromyogr Kinesiol. 2009;19(6): e438-447. doi:10.1016/j.jelekin.2008.09.008

89. Longo UG, Rizzello G, Loppini M, et al. Multidirectional instability of the shoulder: a systematic review. Arthroscopy. 2015;31(12):2431-2443. doi:10.1016/j.arthro.2015.06.006

90. Jacobson ME, Riggenbach M, Wooldridge AN, Bishop JY. Open capsular shift and arthroscopic capsular plication for treatment of multidirectional instability. Arthroscopy. 2012;28(7):1010-1017. doi:10.1016/j.arthro.2011.12.006

91. Xu Y, Wu K, Ma Q, et al. Comparison of clinical and patient-reported outcomes of three procedures for recurrent anterior shoulder instability: arthroscopic Bankart repair, capsular shift, and open Latarjet. J Orthop Surg Res. 2019;14(1):326. doi:10.1186/s13018-019-1340-5

92. Nzeako O, Bakti N, Bawale R, Singh B. Bone block procedures for glenohumeral joint instability. J Clin Orthop Trauma. 2019;10 (2):231-235. doi:10.1016/j.jcot.2018.10.002

93. DiMaria S, Bokshan SL, Nacca C, Owens B. History of surgical stabilization for posterior shoulder instability. JSES Open Access. 2019;3(4):350-356. doi:10.1016/j.jses.2019.08.008

94. Antosh IJ, Tokish JM, Owens BD. Posterior shoulder instability. Sports Health. 2016;8(6):520-526. doi:10.1177/1941738116672 446 
95. Arner JW, Elrick BP, Nolte PC, Goldenberg B, Dekker TJ, Millett PJ. Posterior glenoid augmentation with extra-articular iliac crest autograft for recurrent posterior shoulder instability. Arthrosc Tech. 2020;9 (9):e1227-e1233. doi:10.1016/j.eats.2020.04.023

96. Smith T, Goede F, Struck M, Wellmann M. Arthroscopic posterior shoulder stabilization with an iliac bone graft and capsular repair: a novel technique. Arthrosc Tech. 2012;1(2):e181-185. doi:10.10 16/j.eats.2012.07.003

97. Kraus N, Amphansap T, Gerhardt C, Scheibel M. Arthroscopic anatomic glenoid reconstruction using an autologous iliac crest bone grafting technique. J Shoulder Elbow Surg. 2014;23 (11):1700-1708. doi:10.1016/j.jse.2014.03.004

98. Scheibel M, Kraus N, Diederichs G, Haas NP. Arthroscopic reconstruction of chronic anteroinferior glenoid defect using an autologous tricortical iliac crest bone grafting technique. Arch Orthop Trauma Surg. 2008;128(11):1295-1300. doi:10.1007/ s00402-007-0509-2

99. Taverna E, D’Ambrosi R, Perfetti C, Garavaglia G. Arthroscopic bone graft procedure for anterior inferior glenohumeral instability. Arthrosc Tech. 2014;3(6):e653-660. doi:10.1016/j.eats.2014.08.002

100. Hassebrock JD, Starkweather JR, Tokish JM. Arthroscopic technique for bone augmentation with suture button fixation for anterior shoulder instability. Arthrosc Tech. 2020;9(1):e97-e102. doi:10.1016/j.eats.2019.09.016

101. Abrams JS. Arthroscopic surgical stabilization of glenohumeral dislocations with clavicular graft and remplissage. JBJS Essent Surg Tech. 2019;9(1):e11. doi:10.2106/JBJS.ST.17. 00072
102. Frank RM, Shin J, Saccomanno MF, et al. Comparison of glenohumeral contact pressures and contact areas after posterior glenoid reconstruction with an iliac crest bone graft or distal tibial osteochondral allograft. Am J Sports Med. 2014;42 (11):2574-2582. doi:10.1177/0363546514545860

103. Gilat R, Wong SE, Lavoie-Gagne O, et al. Outcomes are comparable using free bone block autografts versus allografts for the management of anterior shoulder instability with glenoid bone loss: a systematic review and meta-analysis of "The NonLatarjet”. Knee Surg Sports Traumatol Arthrosc. 2021;29 (7):2159-2174. doi:10.1007/s00167-020-06194-z

104. Amar E, Konstantinidis G, Coady C, Wong IH. Arthroscopic treatment of shoulder instability with glenoid bone loss using distal tibial allograft augmentation: safety profile and short-term radiological outcomes. Orthop J Sports Med. 2018;6 (5):2325967118774507. doi:10.1177/2325967118774507

105. Gilat R, Haunschild ED, Lavoie-Gagne OZ, et al. Outcomes of the latarjet procedure versus free bone block procedures for anterior shoulder instability: a systematic review and meta-analysis. Am J Sports Med. 2021;49(3):805-816. doi:10.1177/0363546520925833

106. Meuffels DE, Schuit H, van Biezen FC, Reijman M, Verhaar JA. The posterior bone block procedure in posterior shoulder instability: a long-term follow-up study. J Bone Joint Surg Br. 2010;92 (5):651-655. doi:10.1302/0301-620X.92B5.23529

107. Schwartz DG, Goebel S, Piper K, Kordasiewicz B, Boyle S, Lafosse L. Arthroscopic posterior bone block augmentation in posterior shoulder instability. J Shoulder Elbow Surg. 2013;22 (8):1092-1101. doi:10.1016/j.jse.2012.09.011

\section{Publish your work in this journal}

Open Access Journal of Sports Medicine is an international, peerreviewed, open access journal publishing original research, reports, reviews and commentaries on all areas of sports medicine. The manuscript management system is completely online and includes a very quick and fair peer-review system. Visit http://www.dovepress. com/testimonials.php to read real quotes from published authors. 